\title{
The effect of HIV status on the clinical picture of leprosy: a prospective study in Ethiopia
}

\author{
SHIBRU GEBRE*, PAUL SAUNDERSON*, \\ TSEHAYNESH MESSELE ${ }^{* *}$ \& PETER BYASS ${ }^{+}$ \\ *ALERT, PO Box 165, Addis Ababa, Ethiopia \\ ${ }^{* *}$ Ethiopian Health and Nutrition Research Institute, PO Box 1242, \\ Addis Ababa, Ethiopia \\ ${ }^{+}$School of Community Health Science, Nottingham University, Not- \\ tingham, UK and Department of Public Health and Clinical Medi- \\ cine, Umeå University, Umeå, Sweden
}

Accepted for publication 16 June 2000

Summary No major interaction between HIV infection and leprosy has been documented. The ALERT MDT Field Evaluation Study (AMFES) has allowed the examination of possible interactions in a prospective manner, although the total number of HIV-positive individuals was not high at 22 (3.8\%) of 581 patients tested. There was an excess number of deaths in the HIV-positive group: $27 \%$ compared with $5.7 \%$ in the HIV-negative group, although the causes of death were not recorded (relative risk $4 \cdot 8 ; 95 \%$ CI 2.2-10.2). HIV-positive individuals had a higher risk of ENL reactions (relative risk 5.2; 95\% CI 1.7-15.9). Reversal reactions and neuritis (both acute and chronic) were not significantly influenced by HIV status, although there was a possible increase in recurrent reversal reactions in HIV-positive cases (relative risk 2.2; 95\% CI 0.98-4.7). There was no evidence to suggest an increased risk of developing leprosy or of developing multibacillary rather than paucibacillary disease. There was no association between HIV positivity and the development of impairment.

\section{Introduction}

Infection with the human immunodeficiency virus (HIV) has had a major effect on many infectious diseases, notably tuberculosis (TB) and other mycobacterial diseases. Because of this, epidemiologists and clinicians have been expecting to see changes in the presentation and clinical course of leprosy. In general, no major interaction has been documented. ${ }^{1-3}$

Because the cellular immune response of a patient determines the type of leprosy that will develop, it was also expected that the lepromatous form of the disease may predominate in

Correspondence to: P. Saunderson, ALM, 1 ALM Way, Greenville SC 29601, USA (e-mail: psaunderson@ leprosy.org) 
HIV-positive individuals. ${ }^{4}$ Some reports from East Africa suggest a weak association between HIV positivity and multibacillary (MB) leprosy, ${ }^{5,6}$ while another, from Tanzania, suggests an association between HIV positivity and leprosy (odds ratio $2 \cdot 5$; $95 \%$ CI $2 \cdot 0-3 \cdot 2$ ), but no association with classification. ${ }^{7}$ Studies in Malawi, ${ }^{8}$ South India ${ }^{9}$ and Brazil ${ }^{10}$ found no association between HIV infection and leprosy, although the overall prevalence of HIV infection was very low in the Indian study.

Various authors have examined HIV positivity as a risk factor for the complications of leprosy. A study in Uganda found that type 1 reactions with neuritis were more common in MB patients who were HIV positive, although the study only included 12 HIV-positive and 40 HIV-negative MB cases. ${ }^{11}$ Generally the response to both anti-leprosy and anti-reaction treatment has been found to be satisfactory, ${ }^{11,12}$ although a study in Zambia of eight HIVpositive and $34 \mathrm{HIV}$-negative patients with leprosy found that neuritis was equally common in both groups, but that the outcome of treatment with steroids was poorer in the HIV-positive group. ${ }^{13}$

Erythema nodosum leprosum (ENL) reactions were not reported in HIV-positive individuals until recently and it has been suggested that HIV infection may decrease the risk of this complication. ${ }^{14}$ Case reports of ENL in HIV-positive patients have, however, started to appear. ${ }^{15,16}$

Not surprisingly, HIV infection is associated with an increased death rate amongst leprosy patients. ${ }^{17}$

A long-term prospective study of leprosy in Ethiopia, designed to look at relapses and leprosy reactions, has given an opportunity to study possible interactions with HIV infection.

\section{Materials and methods}

The ALERT MDT Field Evaluation Study (AMFES) recruited 660 patients between 1988 and 1993. There were 10 exclusions due to incorrect diagnosis or incorrect enrolment procedures. Of the 650 patients included, 56 were relapse cases after dapsone monotherapy and 594 were new cases. All were treated with fixed-duration multiple drug therapy (MDT), as recommended by WHO. The research protocol involved taking a serum sample from each patient every year, although for logistic reasons fewer samples were in fact taken. Patients who died were not seen by the leprosy control staff at the time of death and as no procedure for registering deaths exists in the area where the study was carried out, the causes of death are not known.

In all, $581(89 \%)$ patients had their HIV status determined. HIV testing was performed according to a previously evaluated and recommended testing algorithm, ${ }^{18,19}$ which is based on the use of a combination of two different ELISA systems and a rapid assay. Samples were first tested using the Vironistika ELISA kit (Organon Teknika, Boxtel, Holland) and reactive samples were re-tested by Wellcozyme ELISA kit (Murex Diagnostics, Dartford, UK). A third assay which is a rapid test (HIV-SPOT, Genelabs Diagnostics, Singapore) was used for those sera which gave discrepant results in the first two ELISAs. All tests are sensitive for HIV-1 and HIV-2. The testing was done in early 1996, using the most recent serum sample from each patient. Two hundred and thirty-nine $(41 \%)$ of the samples dated from 1988-1991, while 342 (59\%) dated from 1992-1995. For those patients found to be positive, an attempt was made to test previous samples, to indicate, if possible, when they became HIV-positive. 
Patients were reviewed regularly during MDT and 6-monthly thereafter. The skin lesions were examined for signs of reactions and nerve function was tested according to a routine protocol, using voluntary muscle testing (VMT) and sensory testing (ST) with a 10 g nylon monofilament, at 10 points on each hand and foot. The results were recorded and the WHO Impairment Grade (range 0-2) was noted for each eye, hand and foot. The EHF score (or Eye-Hand-Foot score), which is a summation of the six WHO Impairment Grades (and therefore has a range of $0-12),{ }^{20}$ can be calculated from the record of any review. Reversal reactions were defined as the occurrence of signs of inflammation in known leprosy skin lesions, while ENL reactions were defined as the presence of typical ENL skin lesions, away from the known leprosy skin lesions.

Neuritis was defined as the presence of new loss of function in a particular nerve, detected by VMT and ST examinations, or the presence of pain or tenderness in the nerve. The recent loss of one point on a three-point VMT scale or more than one point of sensory loss was taken as indicating active neuritis. Neuritis was said to be chronic if it recurred within 3 months of stopping steroids, or if the standard course of steroids had to be prolonged because of continuing signs and symptoms. Neuritis recurring after a gap of at least 3 months, during which the patient had no symptoms of neuritis and was not being treated with any antireaction medication, was termed recurrent neuritis.

Data analysis was carried out using Epilnfo v6.

\section{Results}

Twenty-two (3.8\%) of the 581 patients were HIV positive, including nine $(3.6 \%)$ of 253 paucibacillary (PB) cases and $13(4.0 \%)$ of $328 \mathrm{MB}$ cases. Six $(27 \%)$ are known to have died, compared with $32(5 \cdot 7 \%)$ HIV negative patients: relative risk $=4.8(95 \%$ CI $2 \cdot 2-10 \cdot 2)$. Female patients were more likely to be HIV positive than males: relative risk $=1.9(95 \% \mathrm{CI}$ 1.3-2.6). Although there are no matched control data, the Ministry of Health estimated the prevalence of HIV infection in Ethiopian adults to have been $3 \cdot 2 \%$ in $1993 .^{21}$

Ten of the $22 \mathrm{HIV}$-positive cases had previous samples that were tested. In two, the previous samples were also positive, but in eight cases the previous samples were negative, indicating seroconversion after diagnosis, during the period 1989-1994.

Table 1 shows the effect of HIV infection as a risk factor for various complications of leprosy. HIV infection was not associated with the development of reversal reactions as such, either for all patients or for MB and PB patients separately. There was, however, a possible association between being HIV positive and having more than one episode of reversal reaction (relative risk: 2.2; 95\% CI: 0.98-4.7). Four of the five HIV-positive patients who had $\mathrm{RR}$, including all three with recurrent reactions, subsequently died, suggesting that they may have been in an immunocompromised state during this period.

The 277 patients with one or more episodes of active neuritis can also be divided into those with only one episode (144 cases) and those with recurrent or chronic neuritis (133 cases). HIV status was not a significant risk factor for either of these patterns of neuritis.

Being HIV positive was significantly associated with the development ENL reactions, despite the small numbers involved (relative risk: 5.2; 95\% CI: 1.7-15.9). All three HIVpositive patients with ENL had minimal neuritis, with most recent EHF scores of 0 .

There was no association between HIV positivity and impairment at any stage of the disease: relative risk for an EHF score of more than 2 at diagnosis is 0.6 (95\% CI: $0 \cdot 2-1 \cdot 7)$, at 
Table 1. Characteristics of HIV-positive and HIV-negative individuals $(n=581)$

\begin{tabular}{|c|c|c|c|c|}
\hline Characteristic & $\begin{array}{l}\text { Result } \\
\text { Yes }\end{array}$ & $\begin{array}{l}\text { HIV-positive } \\
\text { cases }\end{array}$ & $\begin{array}{l}\text { HIV-negative } \\
\text { cases }\end{array}$ & $\begin{array}{l}\text { Relative risk } \\
\quad(95 \% \mathrm{CI})\end{array}$ \\
\hline \multirow[t]{5}{*}{ RJ classification } & TT & 0 & 6 & \multirow{3}{*}{$\begin{array}{l}\text { Relative risk for } \\
\text { being lepromatous: }\end{array}$} \\
\hline & BT & 11 & 248 & \\
\hline & BL & 7 & 216 & \\
\hline & LL & 3 & 87 & $0 \cdot 84$ \\
\hline & NL & 1 & 2 & $(0 \cdot 5-1 \cdot 3)$ \\
\hline \multirow[t]{2}{*}{ WHO classification } & MB & 13 & 315 & $1.05(0.7-1.5)$ \\
\hline & PB & 9 & 244 & - \\
\hline \multirow[t]{2}{*}{ Lepromin test $(n=223)$} & Neg & 6 & 130 & $0.9(0.5-1.5)$ \\
\hline & Pos & 5 & 82 & - \\
\hline \multirow{2}{*}{ Reversal reaction (RR) } & Yes & 5 & 93 & $1.4(0.6-3 \cdot 0)$ \\
\hline & No & 17 & 466 & - \\
\hline \multirow{4}{*}{$\begin{array}{l}\text { Recurrent reversal reaction } \\
\text { amongst } 98 \text { cases of RR } \\
\text { ENL reaction (MB cases only) }\end{array}$} & Yes & 3 & 26 & $2 \cdot 2(0.98-4 \cdot 7)$ \\
\hline & No & 2 & 67 & - \\
\hline & Yes & 3 & 14 & $5 \cdot 2(1.7-15.9)$ \\
\hline & No & 10 & 301 & - \\
\hline \multirow[t]{2}{*}{ Neuritis - all types } & Yes & 11 & 266 & $1.1(0.7-1.6)$ \\
\hline & No & 11 & 293 & - \\
\hline \multirow{2}{*}{$\begin{array}{l}\text { Chronic or recurrent neuritis } \\
\text { amongst } 277 \text { neuritis cases }\end{array}$} & Yes & 5 & 128 & $0.9(0.28-2.9)$ \\
\hline & No & 6 & 138 & - \\
\hline
\end{tabular}

release from treatment (RFT), $1 \cdot 1(95 \%$ CI: $0 \cdot 4-3 \cdot 2)$ and at 5 years after RFT, $1.4(95 \%$ CI: $0 \cdot 3-5 \cdot 8)$.

\section{Discussion}

This study was not designed to investigate risk factors for the development of leprosy, so does not contribute greatly to the debate as to whether HIV-positive individuals are at greater risk of developing the disease. The prevalence of HIV infection in Ethiopia is much greater in urban areas than in rural areas, so any prevalence figures are very dependent on the mix of the sample population. The AMFES cohort includes patients from both urban and rural areas, but with urban dwellers over-represented as compared with the total population of the country. The fact that eight of the $22 \mathrm{HIV}$-positive individuals became positive after leprosy was diagnosed, adds to the conclusion that there is very little evidence from this study to suggest that HIV-positive individuals are more likely than others to be diagnosed as having leprosy.

The results suggest that HIV positivity is associated with ENL reactions, and possibly recurrent reversal reactions. Only one of the four patients with chronic ENL lasting more than 2 years was HIV positive, however, so HIV status is not a sensitive predictor of who will develop this complication. Neuritis does not seem to be more severe, or different in character, in HIV-positive cases and there is no association between HIV positivity and increased impairment at the start of treatment, at RFT or at 5 years after RFT.

Most episodes of neuritis are thought to be, like reversal reactions, the result of cellmediated, delayed type hypersensitivity, so may be expected to be reduced in HIV-positive individuals. However, it is known that the local cellular accumulation and differentiation in HIV-1-infected BT patients in response to infection with $M$. leprae are not impaired, as 
compared with HIV-negative individuals. ${ }^{4}$ ENL reactions, on the other hand, are thought to be due to circulating immune complexes and why these should be more prevalent in HIVpositive cases remains unclear. It is interesting to note that the dramatic increase in cutaneous disease and adverse drug reactions reported in HIV-positive individuals, for example, in patients with tuberculosis who react to the drug thiacetazone, is similarly unexplained, but presumed to be immunologically mediated. ${ }^{22,23}$ These complications also show a worsening as immune function deteriorates. ${ }^{24}$ It is known that, while CD4 lymphocytes are depleted as HIV disease progresses, cytotoxic CD8 cells are significantly increased in number, ${ }^{25}$ including various specific subgroups currently being investigated. ${ }^{26,27}$ Findings such as these may eventually help to explain some of this paradoxical over-expression of some types of immunological activity.

The findings suggest that HIV positivity is not a useful predictor of who will develop leprosy complications, but the increasing prevalence of HIV infection means that more people affected by leprosy may develop these late complications, which require time and skill to manage effectively. This is a further indication of the need to maintain expertise in the management of leprosy and its complications where it continues to exist, even if the prevalence is low. This study suggests that the response to treatment of leprosy and its complications is not impaired by HIV infection.

In summary, this study supports the conclusion that there is no major interaction between HIV infection and leprosy, although some of the known, immunologically mediated complications of leprosy may be more evident in HIV-positive individuals.

\section{Acknowledgements}

We thank the staff of the ALERT Leprosy/TB Control Division for their dedication and perseverance in managing the patients and collecting data over so many years. We thank Mr Wandesan Sime for carrying out the HIV testing and Professor Morten Harboe for helpful comments on the manuscript. The financial support of ILEP, through Netherlands Leprosy Relief (NLR), has been constant throughout the 12 years of the study and is gratefully acknowledged. We also thank ALERT as a whole for institutional and administrative support.

\section{References}

${ }^{1}$ Lucas SB. Human immunodeficiency virus and leprosy. Lepr Rev, 1993; 64: 97-103.

${ }^{2}$ Lienhardt C, Kamate B, Jamet P et al. Effect of HIV infection on leprosy: a three-year survey in Bamako, Mali. Int J Lepr, 1996; 64: 383-391.

${ }^{3}$ Kawuma HJ, Bwire R, Adatu-Engwau F. Leprosy and infection with the human immunodeficiency virus in Uganda: a case-control study. Int J Lepr, 1994; 62: 521-526.

4 Sampaio EP, Caneshi JRT, Nery JA et al. Cellular immune response to Mycobacterium leprae infection in HIVinfected individuals. Infect Immun, 1995; 63: 1848-1854.

5 Orege PA, Fine PEM, Lucas SB et al. A case control study on human immunodeiciency virus-1 (HIV-1) infection as a risk factor for tuberculosis and leprosy in Western Kenya. Tuber Lung Dis, 1993; 74: 377-381.

6 Borgdorff MW, van den Broek J, Chum HJ et al. HIV-1 infection as a risk factor for leprosy: a case-control study in Tanzania. Int J Lepr, 1993; 61: 556-562.

7 van den Broek J, Chum HJ, Swai R, O'Brien RJ. Association between leprosy and HIV infection in Tanzania. Int J Lepr, 1997; 65: 203-210.

${ }^{8}$ Ponnighaus JM, Mwanjasi LJ, Fine PEM et al. Is HIV infection a risk factor for leprosy? Int J Lepr, 1991; 59: 221-228. 
9 Sekar B, Jayasheela M, Chattopadhya D et al. Prevalence of HIV infection and high-risk characteristics among leprosy patients of South India: a case-control study. Int J Lepr, 1994; 62: 527-531.

10 Andrade VL, Avelleira JC, Marques A, Vianna FR. Leprosy as a cause of false positive results in serological assays for the detection of antibodies to HIV-1. Int J Lepr, 1991; 59: 125-126.

11 Bwire R, Kawuma HJ. Type 1 reactions in leprosy, neuritis and steroid therapy: the impact of the immunodeficiency virus. Trans $R$ Soc Trop Med Hyg, 1994; 88: 315-316.

12 Arunthathi S, Ebenezer L, Kumuda C. Reversal reaction, nerve damage and steroid therapy in three multibacillary HIV positive patients. Lepr Rev, 1998; 69: 173-177.

13 Vreeburg AEM. Clinical observations on leprosy patients with HIV-1 infection in Zambia. Lepr Rev, 1992; 63: 134-140.

14 Miller RA. Leprosy and AIDS: a review of the literature and speculations on the impact of CD4+ lymphocyte depletion on immunity to Mycobacterium leprae. Int J Lepr, 1991; 59: 639-644.

15 Olivares LM, Pizzariello GEA, Benetucci J et al. Lepromatous leprosy and HIV infection. Int J Lepr, 1994; 62: 295-296.

16 de Almeida AM, Roselino AMF, Foss NT. Leprosy and HIV infection. Int J Lepr, 1994; 62: 133-135.

17 van den Broek J, Mfinanga S, Moshiro C et al. Survival of HIV-positive and HIV-negative leprosy patients in Mwanza, Tanzania. Int J Lepr, 1998; 66: 53-56.

18 Urassa WK, Breberg-Raden U, Mbena E et al. Alternative confirmatory strategies in HIV-1 antibody testing. J Acquir Immune Defic Syndr, 1992; 5: 170-176.

19 van der Groen G, van Kerckhoven I, Vercauteren G, Piot P. Simplified and less expensive confirmatory HIV testing. Bull World Health Org, 1991; 69: 747-752.

20 van Brakel WH, Reed NK, Reed DS. Grading impairment in leprosy. Lepr Rev, 1999; 70: 180-188.

21 Anon. AIDS in Ethiopia: background, projections, impacts and interventions. Epidemiology \& AIDS Department, Ministry of Health, Addis Ababa, 2nd edn, 1998

22 Okwera A, Johnson JL, Vjecha MJ et al. Risk factors for adverse drug reactions during thiacetazone treatment of pulmonary tuberculosis in human immunodeficiency virus infected adults. Int J Tuberc Lung Dis, 1997; 1: 441-445.

${ }^{23}$ Haslett P, Tramontana J, Burroughs M et al. Adverse reactions to thalidomide in patients infected with human immunodeficiency virus. Clin Infect Dis, 1997; 24: 1223-1227.

24 Coopman SA, Johnson RA, Platt R, Stern RS. Cutaneous disease and drug reactions in HIV infection. $N$ Engl J Med, 1993; 328: 1670-1674.

25 Meyaard L, Otto S, Jonker R et al. Programmed death of T cells in HIV-1 infection. Science, 1992; 257: $217-219$.

${ }^{26}$ Lewis D, E., Yang L, Luo W et al. HIV-specific cytotoxic T lymphocyte precursors exist in a CD28-CD8+ T cell subset and increase with loss of CD4 T cells. AIDS, 1999; 13: 1029-1033.

27 Weekes MP, Wills MR, Mynard $\mathrm{K}$ et al. Large clonal expansions of human virus-specific memory cytotoxic $\mathrm{T}$ lymphocytes within the CD57+ CD28-CD8+ T cell population. Immunology, 1999; 98: 443-449. 